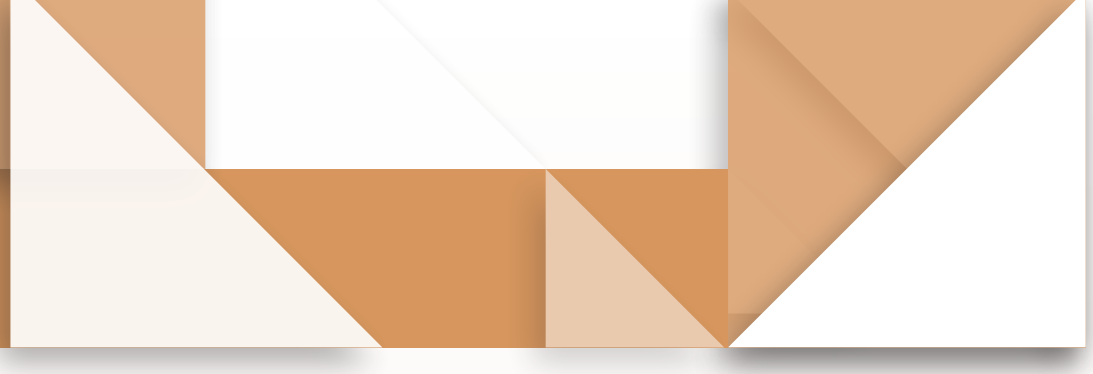

\title{
Concepciones acerca de la
} Tecnología de estudiantes nóveles de carreras científicotecnológicas en Argentina
- Conceptions about the Technology of New Students of Scientific-technological Careers in Argentina

- Concepções sobre a tecnologia de novos estudantes de carreiras científicotecnológicas na Argentina

\section{Resumen}

Este trabajo presenta el análisis de la opinión de alumnos nóveles de las carreras de licenciatura en Biotecnología e Ingeniería en Alimentos de la Universidad Nacional de Quilmes (Argentina) — UNQ - , acerca de diversos aspectos de la Tecnología (T). Se utilizó como instrumento el Cuestionario de Opiniones sobre Ciencia, Tecnología y Sociedad cocts (Vázquez et al., 2006) y se analizaron las respuestas con métodos estadísticos y no estadísticos. Esta metodología presenta variaciones respecto a las que habitualmente se utilizan al aplicar dicho cuestionario. El estudio mostró que los estudiantes poseen visiones sobre la $T$, que están alejadas de las sostenidas por los especialistas. Incluso, se detectaron incoherencias dentro de las mismas opiniones, lo que podría indicar la falta de un modelo mental coherente acerca del fenómeno. En cuanto a las propuestas metodológicas, resultaron útiles para mostrar comportamientos que los métodos anteriores no evidenciaban. Se observó una carencia de los componentes esenciales de la alfabetización científica en alumnos que han elegido una carrera científico tecnológica (Biotecnología, Ingeniería en Alimentos).

Palabras claves

Didáctica de la Tecnología; Educación en Tecnología; CTS; cocts

\section{Diana Inés Roncaglia* Luis Federico Veiga* *}

Doctora en Ciencias Químicas (orientación fisicoquímica), Docente investigador y profesora titular, Grupo GIECIEN (Grupo de Investigación en Enseñanza de las Ciencias)-Departamento de Ciencia y Tecnología, Universidad Nacional de Quilmes, Argentina.

Correo: diana.roncaglia@unq.edu.ar

* * Licenciado en Biotecnología, Becario de posgrado CONICET y Profesor instructor, Grupo GIECIEN IGrupo de Investigación en Enseñanza de las Ciencias)Departamento de Ciencia y Tecnología, Universidad Nacional de Quilmes, Argentina. Correo: luis.veiga@unq.edu.ar

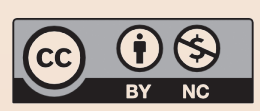

Segundo semestre de 2020 / ISSN 2665-3184

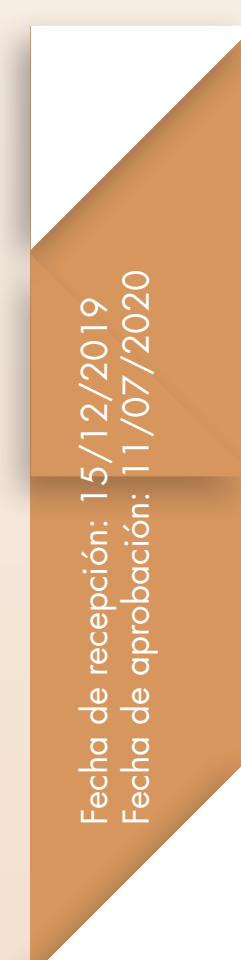

pp. $111-125$ 


\section{Abstract}

This research paper presents an analysis of the opinion of new students of the undergraduate programs in Biotechnology and Food Engineering of the National University of Quilmes (Argentina) - UNQ-, about various aspects of Technology (T). The cocts test (Vázquez et al., 2006) was used as an instrument and analyzing the answers were analyzed with statistical and non-statistical methods. This methodology presents variations with respect to those normally used when applying said test. The study showed that students have visions about $T$ that are far from those held by specialists. Even inconsistencies were detected within the same opinions, which could indicate the lack of a coherent mental model about the phenomenon. As for the methodological proposals, they were useful to show behaviors that the previous methods did not show. A lack of the essential components of scientific literacy was observed in students who have chosen a technological scientific career (Biotechnology, Food Engineering).

Keywords

Technology Teaching; Technology Education; STS; cocts

\section{Resumo}

Este artigo de pesquisa apresenta a análise da opinião de novos alunos dos programas de graduação em Biotecnologia e Engenharia de Alimentos da Universidade Nacional de Quilmes (Argentina) - UNQ - sobre Tecnologia (T). O questionário cocts (Vázquez et al., 2006) foi utilizado como instrumento e as respostas foram analisadas com métodos estatísticos e não estatísticos. Essa metodologia apresenta variações em relação àquelas normalmente utilizadas na aplicação do referido questionário. $\bigcirc$ estudo mostrou que os alunos têm visões sobre a T que estão longe das realizadas por especialistas. Até inconsistências foram detectadas nas mesmas opiniões, o que poderia indicar a falta de um modelo mental coerente sobre o fenômeno. Quanto às propostas metodológicas, elas foram úteis para mostrar comportamentos que os métodos anteriores não mostraram. A carência dos componentes essenciais da literacia científica foi observada nos alunos que opłaram pela carreira científica tecnológica (Biotecnologia, Engenharia de Alimentos).

Palavras-chave

Ensino de Tecnologia; Educação Tecnológica; cTs; cocts 


\section{Introducción}

En el ámbito lberoamericano la Didáctica de la Ciencia ha logrado instalarse en la comunidad de investigación como un área consolidada, así como en una necesidad dentro de los diseños curriculares. En contraposición, el afianzamiento de la Didáctica de la Tecnología, DdT, no ha corrido igual suerte. Si bien dentro de la comunidad de investigadores en didáctica de las ciencias se trata de fomentar la educación en Ciencia Tecnología y Sociedad (CTS), en la práctica, la Tha sido relegada a un lugar marginal. Esto lo enfatiza Layton (Layton, 1988) al indicar que se trata de abordajes CTS. Este abandono no es exclusivo de los investigadores, sino que abarca a todo el espectro de la comunidad educativa, desde las planificaciones hasta los libros (Díaz et al., 2003; Gauchía et al., 2013 1. Sumado a esto, se ha notado que el espacio ganado en el pasado ha mostrado un franco retroceso en las últimas décadas (de Vries, 2018). Debido a esta notable desigualdad entre ciencia (C) y tecnología (T)' que se presenta en el ámbito académico, se indagó acerca del estado de situación la UNQ.

\section{Marco conceptual}

Ante el descuido de la Enseñanza en Tecnología, EeT, es lícito y necesario preguntarse por su origen. Algunos autores vinculan este fenómeno con un menosprecio por el conocimiento práctico en relación con el teórico que sería heredado de la Grecia clásica (Gordillo y Galbarte, 2002). Lo que habría llevado a la concepción de la EeT como una "opción educativa de segunda clase, propia de personas de menores niveles de ingreso y aspiraciones o capacidades intelectuales" (Campo, 2003, p. 60). Díaz cita a Lewis (1991), quien afirma que el factor más importante es la escasa madurez de la filosofía de la T para fundamentar la DdT. En su artículo Díaz et al. (2003) cita a Cardwell, quien destaca la omisión de la historia de la técnica y la T, en algunos historiadores de la ciencia, "incluso distinguiendo con ironía y desprecio entre historia de thinkers (pensadores) y de tinkers (mañosos y chapuceros)" (p. 360). Otro factor que no ha sido mencionado en diferentes trabajos es el poco impacto en el número de citas que tienen los artículos de EeT en relación con la Educación en Ciencia. Se puede hacer una primera aproximación para evidenciar esta situación. Al hacer una búsqueda de dos investigadores tradicionales, como son José Antonio Acevedo-Díaz y Daniel Gil Pérez, se encuentra una tendencia. En el caso del primero, entre los diez artículos más citados de su producción, solo uno está dedicado explícitamente a la tecnología len quinta posición con 187 referencias, siendo que el primero tiene 527 y el promedio de los 10 es de 235); mientras que el segundo, no tiene ninguno (el artículo más popular referido explícitamente a tecnología tiene 67 citas, su artículo más popular tiene 1517 y el promedio para el top 10 es de 606). Por lo que cabría preguntarse por la causalidad de este fenómeno: ¿el tema es "poco interesante" y por eso no es citado o porque no es citado pasa a ser "poco interesante"?

Aun si se aceptara que la Eet está descuidada, quedaría por analizar si vale la pena atenderla. En primer lugar, se pueden considerar los aspectos vinculados a la innovación. Institucionalmente, a nivel global la Organización de las Naciones Unidas, ONU (UNCTAD, 2017) destaca la importancia que tendrían los movimientos de innovación de base (del inglés grass-root innovation, que se definen más adelante) en la concreción de las metas de la Agenda para el Desarrollo Sostenible 2030. A nivel local, el plan Argentina Innovadora 2020 (Ministerio de Ciencia y Tecnología, 2012) que, en principio, debería guiar las actuaciones de los participantes del Sistema Nacional de Ciencia y Tecnología argentino, tiene como uno de sus objetivos particulares "impulsar la cultura emprendedora y la innovación". En el interior de las instituciones académicas tradicionales se ha producido un incremento de la preocupación por el instrumental de laboratorio, lo que ha derivado en una serie de proyectos de diseño abiertos y gratuitos (Pearce, 2017). 
Por fuera de los espacios más institucionalizados, en todo el mundo se han desarrollado movimientos de innovación de base. Según Fressoli (2015), estos movimientos son "redes heterogéneas de activistas, científicos, ingenieros y ONGS que buscan experimentar con formas alternativas de producción de conocimiento y procesos de innovación" (Fressoli et al., 2016, p. 1 15). En el caso argentino, se puede citar "el movimiento de tecnologías apropiadas, el movimiento agroecológico, el movimiento de Software Libre y los nuevos espacios de Fabricación digital (fablabs) y makerspaces" (Fressoli, 2015, p. 7). Queda claro que los procesos de innovación han sido centrales tanto en espacios institucionales como no institucionales. La Eet podría hacer distintos aportes a la innovación Londoño (1991) considera que puede ayudar "a fortalecer la capacidad nacional para asimilar y generar tecnología" (p. 7), lo que aportaría a la soberanía de las naciones, sobre todo, aquellas que están en desarrollo. A su vez, otros autores indican que muchos de los esfuerzos que se han hecho por lograr innovaciones de base científica han fracasado por una concepción lineal de la innovación (es decir, una visión que caracteriza a la T como ciencia aplicada) (Bortz y Thomas, 2019). Con lo cual, la Eet podría ayudar en la construcción de una correcta concepción de la Naturaleza de la Tecnología, DdT, por parte de los actores involucrados en estos procesos. Así como volverla más democrática y responsable (Molina, 2011 ; Valdés et al., 2002).

En segundo lugar, desde un punto de vista epistemológico, cierta concepción de alfabetización científica excluiría o deformaría el papel de la tecnología (Cajas, 200 la). La EeT ayudaría no solo a comprender la NdT sino, también, la Ndc (Fernández et al., 2003; Maiztegui et al., 2002). Sumado a esto, autores como Barak y Zadok han observado que este tipo de educación también ayudaría a que los estudiantes valoricen el conocimiento científico (Barak y Zadok, 2009). Además, Cajas destaca que se puede usar la Eet para introducir conceptos (como el de control) que son compartidos por otras áreas distintas que la T, por ejemplo: sistemas de control social, biológico, etc. (Cajas, 2001).

Por último, desde un punto de vista didáctico, una de las principales ventajas de la Eet es su cercanía a las vivencias de los estudiantes. De esta forma, Valdés et al. (2002), indican que permitiría un aprendizaje significativo por parte de los estudiantes, lo cual sería una ventaja sobre la Ndc. Muchas veces se suele apelar a ejemplos de la técnica para acercar la ciencia a la vida cotidiana de los estudiantes, error que denota una clara falla en la concepción de los docentes.

Según Gilbert (1995), el abordaje adoptado se puede distinguir entre la enseñanza para, sobre y en la tecnología. Esta clasificación está hecha con base en un análisis de Pacey (1983) para quien la tecnología tiene tres aspectos: el técnico (vinculado con los conocimientos, capacidades y materiales necesarios), el cultural (valores y criterios subyacentes en la elección de los fines con los que se desarrolla la tecnología y en la evaluación de sus resultados) y el organizativo (la economía y sociología de la tecnología). De este modo, una enseñanza para la tecnología estaría centrada en los aspectos técnicos. La enseñanza sobre la tecnología se enfocaría en la cuestión cultural y organizativa, mientras que una enseñanza en la tecnología trataría de integrar los tres aspectos. Naturalmente, esta última, por su pretensión de dar una visión integral es el ideal a trazar. 
Entonces, si lo que se desea es construir el concepto de $T$, una condición necesaria para educar es contar con un instrumento para evaluar la concepción previa que se tiene de aquella. Los instrumentos que se han utilizado para estos fines en Iberoamérica pueden ser clasificados en sistematizados y específicos. Por sistematizados se alude a aquellos que fueron aplicados por distintos grupos y en distintas condiciones, mientras que por específicos a los que fueron aplicados en reducidas ocasiones y ámbitos.

El instrumento sistematizado empleado en Iberoamérica es el cocts (Bennássar Roig et al., 2010 ; Vázquez et al., 2006) que consiste en un cuestionario tipo Likert (escala 1 a 9) donde se pide al sujeto que califique una serie de afirmaciones según su grado de acuerdo con un Modelo de Respuesta Múltiple (MRM). Luego, los resultados suelen ser presentados con un índice relativo a las opiniones de especialistas consultados por los investigadores que desarrollaron el cuestionario. Este índice se llama Índice Actitudinal Normalizado (IAN), el cual toma valores que van entre -1 (completamente alejado de la opinión de los especialistas) y 1 (coincidencia perfecta con la opinión de los especialistas). Las situaciones cubren un amplio espectro que va desde el vínculo entre tecnología y ciencia hasta asuntos de género. Por tanto, este instrumento no fue desarrollado para evaluar específicamente la cuestión tecnológica, sino que, de forma más general, la NdCyT, vista desde el enfoque CTS.

En algunos de los trabajos, se realiza una aplicación de todo el cocts y luego se analizan las cuestiones vinculadas a la tecnología (Acevedo Díaz et al., 2005; Cadena y Agudelo, 2013 ) y, en otros, se aplican solo las cuestiones vinculadas a la tecnología (Acevedo Díaz et al., 2005; Tessio et al., 2012; Hugo et al, 2012; 2014). Una de las aplicaciones más importantes del cuestionario lo llevó a cabo el Proyecto Iberoamericano de Evaluación de Actitudes relacionadas con la Ciencia, la Tecnología y la Sociedad (PIERACTS). Se trató de una "investigación cooperativa internacional desarrollada por una veintena de investigado- res de siete países iberoamericanos (Argentina, Brasil, Colombia, España, México, Portugal y Panamá)", un análisis de aquellas vinculadas a la cuestión tecnológica, específicamente, las cuestiones 10411, 30111 1,40221,40531, $80131,10211,10421$ y 40211.

En el presente trabajo, se evalúa la opinión de nóveles estudiantes universitarios de carreras científico-tecnológicas respecto a cuestiones vinculadas a la tecnología. El instrumento utilizado fue el cocts. El motivo de la elección fue, principalmente, porque permitiría comparar los resultados con otros datos previos les decir, por haber sido usado de manera sistematizada). Además, nuestro grupo de investigación tiene una larga experiencia con el mismo. El cuestionario no se aplicó completo, sino que se seleccionaron las cuestiones $10211,10421,10431,40211,40411$ y 40521. Las cuestiones 1021 1, 10421 y 40211 fueron evaluadas en el pIERACTS. Frente a las cuestiones, los estudiantes jóvenes de ciencias obtuvieron valores de IAN de entre $-0,05$ y $-0,15,0,075$ y 0,125, y, 0,00 y 0, 10, para 10211 , 10421 y 40211 , respectivamente. Estos valores cercanos a $\mathrm{O}$ significan un ajuste parcial a la opinión de los especialistas.

Las hipótesis de trabajo se basaron en los antecedentes del PIERACTS, donde la concepción de $T$ de los estudiantes fue ingenua. Al término del curso estudiado (cuatrimestral) no se deberían observar cambios significativos debido a que el diseño actual de la asignatura no tiene como objetivo construir el concepto de $T$ con los estudiantes.

\section{Metodología}

De las 100 cuestiones que componen el cocts, se seleccionaron 6 relacionadas a la $T$. Se optó por un número reducido de preguntas para garantizar, por un lado, que el tiempo demandado para completarlo fuera el necesario para que los estudiantes y pudieran responder en clase $y$, por el otro, evitar el tedio debido a la extensión.

La muestra de estudiantes estuvo compuesta por dos grupos aula de segundo año 
de las carreras de Licenciatura en Biotecnología e Ingeniería en Alimentos, del Departamento de Ciencia y Tecnología de la Universidad Nacional de Quilmes durante el segundo cuatrimestre de 2018 y el primero de 2019 para un total de 21 estudiantes, de entre 18 y 22 años de edad.

Se les administró el instrumento de evaluación antes de comenzar el curso (pretest) y al final (pos-test). Los estudiantes fueron ciegos a la experiencia, es decir, no recibieron ninguna pista ni información que les advirtiera que el mismo instrumento volvería a serles aplicado al final del curso. Además, las condiciones temporales de ambos momentos (pre y post) también fueron los mismos, para controlar las potenciales variables ambientales intervinientes; el tiempo transcurrido entre ambos momentos de evaluación es suficientemente amplio (cuatro meses) para evitar la influencia del recuerdo del pretest sobre el postest. Los grupos elegidos fueron equivalentes en las variables contextuales que definen los grupos (pertenencia a la misma institución y al mismo nivel, parecido nivel académico, similar composición de chicos y chicas).

El procesamiento de datos tuvo lugar según se detalla a continuación. En primer lugar, se transformaron los índices de las respuestas ( 1 a 9) en niveles de acuerdo (NDA). Si la valoración estaba entre 1 y 3 , el NDA era I (con un valor numérico de $N D A, N^{\circ} A^{\circ}$, de 1). Si estuviera entre 4 y 6 , sería ॥ $\left(\mathrm{NDA}^{\circ}=2\right)$. Entre 7 y 9 , el NDA sería $\|$ II $\left(N D A^{\circ}=3\right)$. En trabajos de otros autores la estrategia elegida consiste en asignar un IAN (Índice de Acuerdo Normalizado). Sin embargo, se decidió no optar por esta estrategia porque, al presentar los datos, quien los lee está obteniendo una información ya relativizada en función de los especialistas. Esto se da, principalmente, cuando la calificación dada por los especialistas es plausible. En ese caso, si el estudiante da una calificación de 1 ० 9, el IAN informado es de -1, siendo indistinto que el estudiante esté completamente de acuerdo o en total desacuerdo. Por otro lado, no se trabajó con los índices crudos sin transformar, porque el IAN no da información relevante para este tipo de evaluaciones. Lo que se busca es saber si los estudiantes están en desacuerdo, medianamente de acuerdo o totalmente de acuerdo. Es decir, si están en el NDA I, \|I O III y no si, por ejemplo, dentro del NDA II están más cerca del 4 o del 6.

Los datos así transformados fueron analizados de dos formas:

1) Análisis estadístico, empleando el software SPS. A partir de los NDA ${ }^{\circ}$, se calcularon las medias y los desvíos estándar, tanto para el pretest como para el postest. También, se determinó el valor p usando la prueba estadística de Wilcoxon y se tomó como criterio para demarcar variaciones significativas un $p$ (bilateral) $\leq 0,05$. Por último, para determinar la magnitud de la variación, se calculó el tamaño del efecto usando la d de Cohen ( $d \geq 0,5$ efecto alto).

2) Análisis no Estadístico. a) Se calcula el porcentaje de respuestas según el NDA y la variación entre pretest y postest. Además del promedio del NDA ${ }^{\circ}$ y su variación. b) El ajuste entre la opinión de los estudiantes y la de los especialistas y autores. Para ello, se relacionaron las clasificaciones de Ingenua, Plausible y Adecuada con los valores 1, 2 y 3, respectivamente. Luego, se calculó la diferencia entre este valor y el promedio del NDA ${ }^{\circ}$ del postest. Si la diferencia entre ellos es menor o igual a 0,25, se considera que las visiones ajustaron perfectamente. Si está entre 0,25 y 0,75, el ajuste es 
distante. Si es mayor o igual a 0,75, no hay ajuste. También se obtuvo un promedio para todas las oraciones, con lo cual se califica el ajuste general para la cuestión. Finalmente, se calculó un promedio de todas las cuestiones, que dio el ajuste para la cuestión tecnológica.

\section{Resultados}

Inicialmente, se transformaron las respuestas de los cuestionarios al NDA ${ }^{\circ}$, a cuyos valores se aplicó la prueba estadística de Wilcoxon (tabla 1 y 1 bis) para hallar diferencias significativas luego de cursar la asignatura.

Tabla 1. Análisis estadístico de las cuestiones 10421,40211 y 40521

\begin{tabular}{|c|c|c|c|c|c|c|c|}
\hline \multirow{2}{*}{\multicolumn{2}{|c|}{ Pregunta }} & \multirow[t]{2}{*}{$N$} & \multicolumn{2}{|c|}{ Media } & \multicolumn{2}{|c|}{$\begin{array}{c}\text { Desvío } \\
\text { estándar }\end{array}$} & \multirow{2}{*}{$\begin{array}{l}\text { valor } p \\
\text { (bilateral }\end{array}$} \\
\hline & & & PRE & POST & PRE & POST & \\
\hline \multirow{8}{*}{10421} & $A$ & 17 & 1,4 & 1,4 & 0,6 & 0,6 & 1,000 \\
\hline & B & 18 & 1,7 & 1,9 & 0,8 & 0,9 & 0,222 \\
\hline & C & 20 & 3,0 & 2,9 & 0,2 & 0,4 & 0,157 \\
\hline & D & 20 & 2,9 & 2,8 & 0,3 & 0,4 & 0,257 \\
\hline & $E$ & 19 & 2,8 & 2,7 & 0,5 & 0,6 & 0,180 \\
\hline & $\mathrm{F}$ & 20 & 2,2 & 2,0 & 0,7 & 0,9 & 0,285 \\
\hline & $G$ & 20 & 1,9 & 1,5 & 0,9 & 0,7 & 0,013 \\
\hline & $\mathrm{H}$ & 20 & 1,3 & 1,2 & 0,4 & 0,4 & 0,480 \\
\hline \multirow{8}{*}{40211} & $A$ & 20 & 2,7 & 2,4 & 0,6 & 0,8 & 0,141 \\
\hline & B & 21 & 2,7 & 2,5 & 0,6 & 0,7 & 0,206 \\
\hline & C & 21 & 2,7 & 2,6 & 0,6 & 0,7 & 0,317 \\
\hline & D & 21 & 2,6 & 2,8 & 0,5 & 0,6 & 0,248 \\
\hline & $\mathrm{E}$ & 21 & 1,8 & 1,8 & 0,7 & 0,9 & 1,000 \\
\hline & $\mathrm{F}$ & 21 & 1,7 & 1,8 & 0,8 & 0,8 & 0,803 \\
\hline & G & 21 & 1,3 & 1,3 & 0,5 & 0,5 & 1,000 \\
\hline & $\mathrm{H}$ & 21 & 2,1 & 2,2 & 0,8 & 0,9 & 0,512 \\
\hline \multirow{6}{*}{40521} & $A$ & 20 & 2,6 & 2,7 & 0,7 & 0,6 & 0,414 \\
\hline & B & 21 & 2,6 & 2,8 & 0,7 & 0,5 & 0,102 \\
\hline & C & 21 & 2,2 & 2,4 & 0,9 & 0,8 & 0,271 \\
\hline & D & 21 & 2,4 & 2,5 & 0,7 & 0,7 & 0,627 \\
\hline & $\mathrm{E}$ & 21 & 2,6 & 2,1 & 0,7 & 0,8 & 0,021 \\
\hline & $\bar{F}$ & 21 & 2,5 & 2,0 & 0,8 & 0,9 & 0,028 \\
\hline
\end{tabular}

N: el número de estudiantes que respondió y que, además, lo hizo de forma distinta entre uno y otro test. Media y el desvío estándar de los NDA ${ }^{\circ}$ pre y el postest. Valor p para la prueba de los rangos con signos de Wilcoxon.
Se sombrean con gris más oscuros aquellas cuestiones cuyo valor de $p \leq 0,05$, es decir, presentan diferencias significativas para la variación entre pre y postest.

Tabla 1 bis. Análisis estadístico de las cuestiones 10211, 10431, 40411

\begin{tabular}{|c|c|c|c|c|c|c|c|}
\hline \multirow{2}{*}{ Pregunta } & & \multirow[t]{2}{*}{$\mathrm{N}$} & \multicolumn{2}{|c|}{ Media } & \multicolumn{2}{|c|}{$\begin{array}{l}\text { Desvío } \\
\text { estándar }\end{array}$} & \multirow{2}{*}{$\begin{array}{c}\text { valor } p \\
\text { (bilateral) }\end{array}$} \\
\hline & & & PRE & POST & \begin{tabular}{|l} 
PRE \\
\end{tabular} & POST & \\
\hline \multirow{4}{*}{10211} & B & 21 & 2,4 & 2,5 & 0,8 & 0,7 & 0,589 \\
\hline & C & 21 & 2,8 & 2,8 & 0,4 & 0,4 & 1,000 \\
\hline & G & 21 & 2,6 & 2,3 & 0,7 & 0,7 & 0,145 \\
\hline & $\mathrm{H}$ & 21 & 1,6 & 1,4 & 0,7 & 0,7 & 0,492 \\
\hline \multirow{5}{*}{10431} & A & 21 & 1,7 & 1,6 & 0,9 & 0,7 & 0,480 \\
\hline & B & 21 & 2,9 & 2,9 & 0,3 & 0,4 & 0,564 \\
\hline & C & 20 & 2,4 & 2,5 & 0,7 & 0,7 & 0,480 \\
\hline & D & 21 & 2,7 & 2,4 & 0,7 & 0,7 & 0,248 \\
\hline & $\mathrm{E}$ & 21 & 2,7 & 2,8 & 0,6 & 0,4 & 0,527 \\
\hline \multirow{6}{*}{40411} & A & 21 & 2,6 & 2,6 & 0,6 & 0,7 & 1,000 \\
\hline & B & 21 & 2,6 & 2,5 & 0,7 & 0,7 & 0,627 \\
\hline & C & 21 & 2,1 & 2,3 & 0,8 & 0,8 & 0,163 \\
\hline & D & 21 & 2,9 & 2,8 & 0,4 & 0,4 & 0,705 \\
\hline & E & 21 & 1,9 & 1,7 & 0,9 & 0,8 & 0,334 \\
\hline & $\mathrm{F}$ & 21 & 1,2 & 1,2 & 0,4 & 0,5 & 0,705 \\
\hline
\end{tabular}

Una vez determinados los enunciados en las cuales hubo diferencia significativa, se calculó la d de Cohen para cuantificar el tamaño del efecto (tabla 2).

Tabla 2. Análisis estadístico del tamaño
del efecto (d de Cohen) para las
cuestiones 10421G y 40521E y F
\begin{tabular}{|r|c|}
\hline Pregunta & d \\
\hline 10421 G & 0,50 \\
\hline 40521 E & 0,67 \\
\hline F & 0,59 \\
\hline
\end{tabular}

Análisis del tamaño del efecto para las cuestiones que presentaron diferencias estadísticas significativas (10421 G, 40521 E y F). 
Luego se realizaron dos análisis no estadísticos. Con el primero, se buscaba conocer la distribución de las respuestas según el NDA y su variación tras el período que duró la cursada (Tabla 3 y 3 bis).

Tabla 3. Análisis no estadístico I cuestiones 10421,4021 1,40521

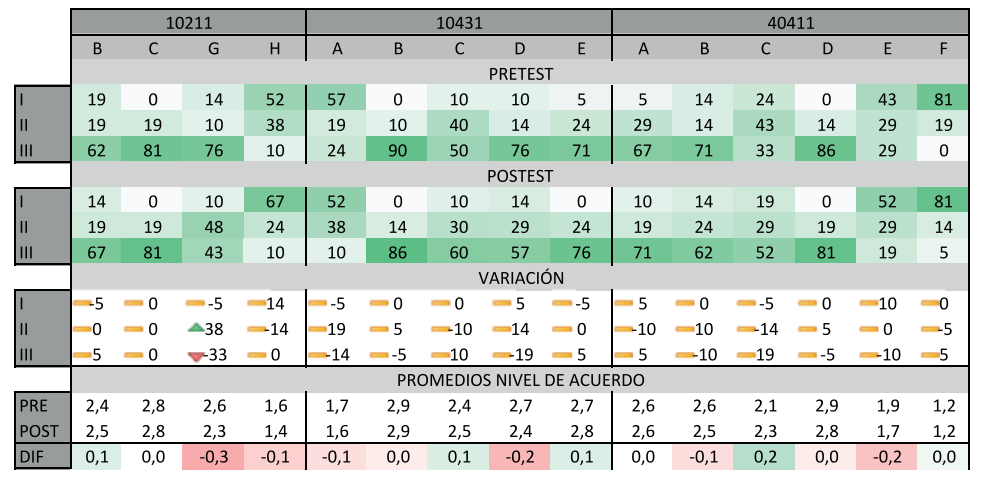

Resultados de las cuestiones 10421, 40211 y 40521 del test de cocts. La tabla está ordenada: 1) con el porcentaje de respuestas desagregado por NDA ( corresponde a las calificaciones de 1, 2 y 3; ॥ a 4, 5 y 6; || a 7, 8 y 9) y, 2) con el promedio del NDA ${ }^{\circ}$. Cada una de ellas, a su vez, presenta los resultados de pretest, postest y variación entre ambas instancias. El código de color utilizado varía. Para el porcentaje de respuestas en pretest como para el de postest, la intensidad del color verde aumenta con el valor del porcentaje. Para la variación de porcentaje, si es menor a -20, se simboliza con una flecha roja hacia abajo; si está entre -20 y 20, se indica con una línea amarilla; si es mayor a 20 , se denota con una flecha verde hacia arriba. En la diferencia de promedios del NDA ${ }^{\circ}$, el color varía desde un rojo intenso para los más negativos hasta un verde intenso para los más positivos.

\section{Tabla 3bis. Análisis no estadístico I cuestiones 10221, 10431, 40411}

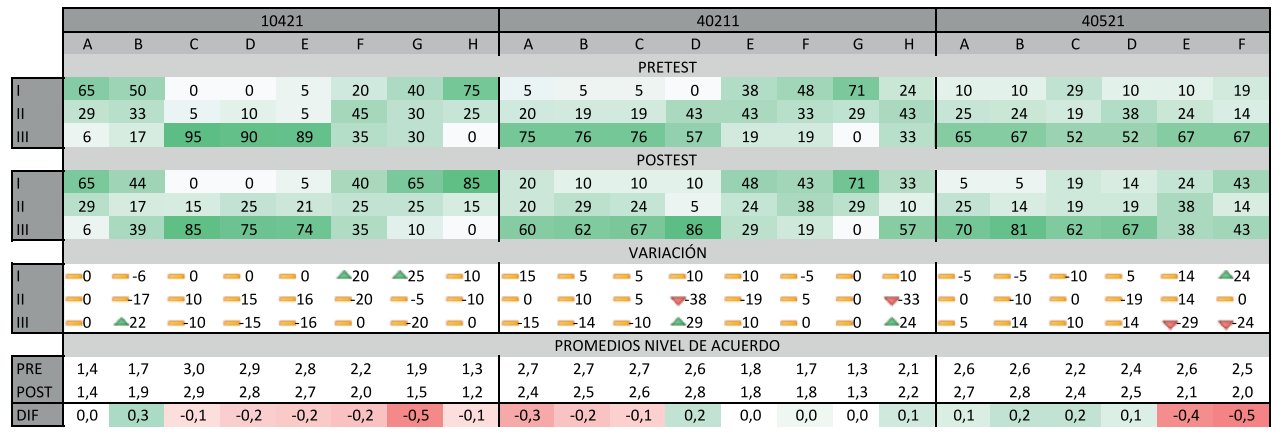

Resultados de las cuestiones 1021 1, 10431 y 40411 del test de cocts. Leer referencias de Tabla 3.

Con el segundo, se pretendió evidenciar el grado de adecuación entre la visión de los estudiantes y las de los especialistas y los autores del artículo (Tabla 4 y 4 bis). 


\begin{tabular}{|c|c|c|c|c|c|c|c|c|c|c|c|c|c|c|c|}
\hline & \multicolumn{3}{|c|}{10211} & \multicolumn{5}{|c|}{10431} & \multicolumn{6}{|c|}{40411} \\
\hline & & $\mathrm{C}$ & G & $\mathrm{H}$ & $\mathrm{A}$ & $B$ & $\mathrm{C}$ & $\mathrm{D}$ & $E$ & $\mathrm{~A}$ & $B$ & $\mathrm{C}$ & $\mathrm{D}$ & $E$ & $\mathrm{~F}$ \\
\hline & ESTUDIANTES & \begin{tabular}{|l|l|}
2,5 & 2,8 \\
\end{tabular} & 2,3 & 1,4 & 1,6 & 2,9 & 2,5 & 2,4 & 2,8 & 2,6 & 2,5 & 2,3 & 2,8 & 1,7 & 1,2 \\
\hline \multirow{6}{*}{$\begin{array}{l}\underset{c}{\check{n}} \\
\underset{m}{m}\end{array}$} & ESPECIALISTAS & $\bar{P}$ & A & $P$ & $P$ & $\mathrm{~A}$ & $P$ & $\mathrm{~T}$ & 1 & $P$ & $\mathrm{~A}$ & $\mathrm{~A}$ & $P$ & I & $\mathrm{I}$ \\
\hline & $\%$ POR CATEGORÍA & $0,0 \quad 50,0$ & 50,0 & & & 20,0 & 40,0 & 40,0 & & & 16,7 & 66,7 & 16,7 & & \\
\hline & $\begin{array}{l}\text { PROMEDIO } \\
\text { PONDERADO }\end{array}$ & \multicolumn{3}{|c|}{0,9} & \multicolumn{5}{|c|}{0,9} & \multicolumn{6}{|c|}{0,6} \\
\hline & AUTORES & $P$ & A & $\mathrm{P}$ & A & $\mathrm{P}$ & $T$ & $\mathrm{I}$ & I & $P$ & $P$ & A & $\mathrm{T}$ & $\mathrm{I}$ & $\mathrm{T}$ \\
\hline & $\%$ POR CATEGORÍA & $0,0 \quad 50,0$ & 50,0 & & 0,0 & 0,0 & \multicolumn{3}{|l|}{100,0} & \multicolumn{6}{|c|}{$\begin{array}{lll}16,7 & 66,7 & 16,7 \\
\end{array}$} \\
\hline & PROMEDIO & \multicolumn{3}{|c|}{0,9} & \multicolumn{5}{|c|}{1,4} & \multicolumn{6}{|c|}{0,7} \\
\hline
\end{tabular}

Promedios de NDA ${ }^{\circ}$ del postest 10211,10431 y 40411 . En una misma fila, la opinión que dan los especialistas, así como el nivel de coincidencia entre esta y la de los estudiantes. La opinión está simbolizada por las letras I Ingenua, P Plausible o A Adecuada. La adecuación se simboliza con el color de la celda. Primero se relaciona la opinión de los especialistas con un valor (I es 1, P es 2 y A es 3). Luego, se compara el promedio de la opinión de los estudiantes con este valor. Si la diferencia es menor o igual a 0,25 , el color es verde. Si está entre 0,25 y 0,75, el color es amarillo.
Si es mayor o igual a 0,75 , el color es rojo. Lo siguiente que se indica es el porcentaje por categoría de ajuste, es decir, qué porcentaje de las oraciones ajustó bien, regular o mal. Finalmente, se presenta el promedio ponderado que se calcula como el promedio de las diferencias entre los estudiantes y la referencia lel valor de esta diferencia no está informado en la tabla) y cuyo color se asigna de igual forma que para la fila ESPECIALISTAS. A continuación, la misma información se presenta en relación a la opinión de los autores del artículo.

Tabla 4bis. Análisis no estadístico || cuestiones 10421, 4021 1, 40521

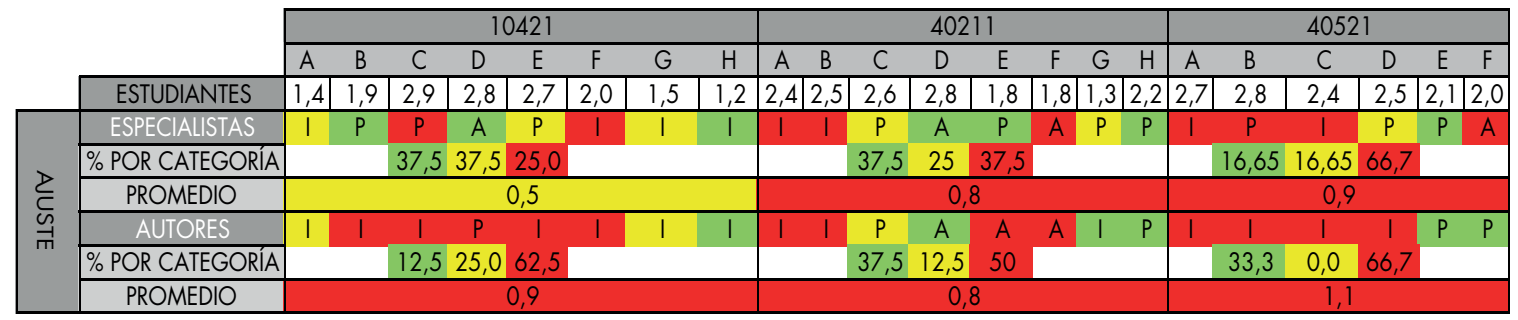

Promedios de $\mathrm{NDA}^{\circ}$ del postest para las cuestiones 10211,10431 y 40411 del test de cocts. (Ver Tabla 4 para referencias).

\section{Discusión}

Al comparar los datos obtenidos aquí con los estudios previos del PIERACTS, los estudiantes jóvenes de ciencia que habían respondido las cuestiones 10211 y 40211 obtuvieron IAN cercanos a 0,0 , mientras que para la cuestión
10421 obtuvieron IAN cercanos a 0, 1. Si bien los IAN no se muestran explícitamente aquí, sí fueron calculados, sus valores para 10211 , 10421 y 40211 fueron de $-0,22,0,19$, $-0,19$ respectivamente. En definitiva, los resultados son similares.

Al analizar la coherencia entre las opiniones, se observa una falla en la concepción que los estudiantes tienen del vínculo entre cyт. Sostienen que la cyt tienen el mismo cuerpo de conocimientos $y$, a su vez, que la T se basa 
tanto en conocimientos científicos como tecnológicos. Además, desestiman la opinión de que, como la cyT comparten el mismo cuerpo de conocimientos, sea igual invertir en una u otra. En resumen, faltan evidencias para definir cuál es su perspectiva en este asunto.

Cuando se confronta el ajuste entre las opiniones de los estudiantes y las referencias es posible afirmar que no hubo un buen ajuste. Esto queda claro si se observa el promedio ponderado de las diferencias de NDA (Tabla 4 y 4 bis). Como panorama general, si se calcula el promedio de los promed

En los ponderados de las diferencias (no está incluido en las tablas) se ve que, en relación a la opinión de los especialistas, es 0,75 (es decir, malo) y, en relación a la de los autores, es 0,98 . En cada cuestión particular, también se ven ajustes entre regulares y malos. Al considerar la opinión de los especialistas, 10211 , 10431, 40211 y 40521 tienen diferencias por encima de 0,75, y 40411 y 10421 tienen diferencias de entre 0,25 y 0,74. Es decir, apenas se acercan a la visión en asuntos de inversión pública en cyт y en lo referido al "impacto" sobre la sociedad. Cuando se toma la intervención de los autores, la situación se repite, solo que 10421 tiene una diferencia mayor a 0,75. De esta forma, los estudiantes parecen tener una visión a penas cercana a la de los autores, solo en lo vinculado a la relación de cyt hacia la sociedad. De forma general, se puede afirmar que el ajuste de los estudiantes fue peor cuando las situaciones estaban vinculadas exclusivamente con la cuestión tecnológica (1021 1, 10431 , 40521 ) y cuando se trata de relaciones de la sociedad hacia la сут (4021 1).

En las variaciones después del curso académico, los análisis estadísticos permiten afirmar que solo hubo cambios en la visión de los estudiantes en relación a tres cuestiones: $10421 \mathrm{G}, 40521 \mathrm{E}$ y F, cambios que fueron tanto positivos como negativos (en relación a los referentes). Esto se evidencia a través de la prueba de Wilcoxon. En los tres casos, el tamaño del efecto fue considerable $(d \geq 0,50)$. Para $10421 \mathrm{G}$, se pasó de una media de 1,9 a 1,5, esta cuestión está definida tanto por los especialistas como por los autores de este trabajo como Ingenua, con lo cual, una disminución de la media resulta un efecto positivo. La cuestión 40521 E disminuyó su valoración de 2,6 a 2,1. La definición que dan tanto los especialistas como los autores es que se trata de una afirmación Plausible. Es decir, que la calificación debería ser cercana a 2. Por lo tanto, la variación fue en el sentido correcto. Finalmente, la cuestión $40521 \mathrm{~F}$ pasó de 2,5 a 2,0. Según los especialistas, esta afirmación es Adecuada, por lo que la variación sería negativa (se aleja del valor máximo de 3). Los autores, en cambio, la consideramos como Plausible, y la variación sería positiva.

Al momento de observar las variaciones en el porcentaje de respuestas por categoría (Tabla 3 y 3 bis), se notan modificaciones en el comportamiento de la población que no son detectados por el método estadístico. Por ejemplo, $10421 \mathrm{~B}$ y F, $40211 \mathrm{D}$ y H y $10211 \mathrm{G}$ presentan variaciones mayores a $20 \%$ en alguno de los niveles. Sin embargo, ninguno de estos cambios es detectado por el método estadístico. Al parecer, lo que sucede es que solo es sensible a "movimientos" entre el NDA I y el NDA III. Para ilustrar este punto, puede compararse 10421 G y 40211 D. En un caso, la variación es de $+25 \%$ para l y $-25 \%$ para $\|$ ll y, en el otro, $-38 \%$ para $\|$ y $+29 \%$ para II. Sin embargo, el método sólo detecta $10421 \mathrm{G}$. Una explicación para esto es que las variaciones entre los NDA I y III provocan grandes modificacio- 
nes de la media (los valores asignados a I y III son más lejanos), mientras que modificaciones de igual magnitud entre niveles contiguos no. En definitiva, si se consideran estas variaciones, habría que sumar los corrimientos de 1042 1B, $40211 \mathrm{D}$ y H y $10211 \mathrm{G}$. La migración de ॥ $(-17 \%)$ a III (+22\%) en 10421 B habría que considerarla negativa, ya que los especialistas la consideran Plausible y los autores Ingenua. También hubo una variación de ॥ (-38\%) a III (+29\%) en 40211D que se puede considerar positiva porque ambas referencias toman a la afirmación como Adecuada. El cambio de ॥ (-33 \%) a III (+24\%), en $40211 \mathrm{H}$, no sería positiva ya que es considerada Plausible por ambas referencias. Tampoco sería deseable el desplazamiento de II (-33\%) a ॥ (+38\%) en $10211 \mathrm{G}$ porque se aleja del rango de Adecuada.

Con este análisis, puede afirmarse que el efecto del curso sobre la opinión de los estudiantes fue casi nulo. Solo hubo variaciones en 7 de las 37 afirmaciones ( 3 estadísticamente significativas y 4 no). Sin perder esto de vista, se puede analizar en qué se vio afectada la visión de los estudiantes. Se ha empobrecido la concepción respecto a qué es la tecnología (pasaje de III a ॥ en $10211 \mathrm{G}$ ). Además, por un lado, los estudiantes parecieran empezar a considerar que ciencia y tecnología no son lo mismo (movimiento de II a I| en 10421 B) e, incluso, hay un abandono de la posición de la ciencia como una actividad pura y la tecnología como algo mundano y vil (movimiento de III a I en $10421 \mathrm{Gl}$; aunque, por el otro, cuando se pregunta explícitamente por este tema, no hay variaciones en la opinión (principalmente, 10431). Es decir, no hay un cambio coherente, posiblemente debido a dos razones: o el instrumento no es capaz de explicitar estos pensamientos o las variaciones no son producto del curso en sí. No se puede descartar ninguna de las dos posibilidades, pero podría tratarse de la segunda, ya que el curso no fue hecho con la intención expresa de alterar la visión de los estudiantes. Respecto a las relaciones CTS, se ha visto una apreciación en la participación de distintos actores en cuestiones controversiales (aumento de III y caída de II en 40211 D), aunque consideran que esto no es válido para todas las controversias (pasaje de ॥ a III en $40211 \mathrm{H}$ ). Finalmente, relacionado al impacto de la alta tecnología en los puestos de trabajo, se abandonó una posición optimista para adoptar una más moderada (pasaje de III a || y I en 40521 E y de II a I en F).

Así y todo, hay que tener en cuenta que este tipo de análisis tiene su dificultad. Resulta poco apropiado atribuir únicamente a la cursada de la materia el cambio de opinión de los estudiantes. Hay muchos otros factores, vinculados a la vida universitaria como a la no universitaria, que están actuando sobre la opinión de los estudiantes. Esto no debe ser perdido de vista nunca.

\section{Conclusiones}

Se puede concluir, en coincidencia con estudios anteriores, que los estudiantes evaluados mostraron opiniones respecto a la T que están alejadas de las de los especialistas. Los cambios después del curso académico no fueron importantes (3 opiniones de las 37 oraciones cambiaron de forma significativa y 4 más con criterios no estadístico) y que, incluso, algunas de las variaciones fueron en sentidos indeseados. En otra perspectiva, se vio que los estudiantes sostuvieron opiniones paradójicas acerca de la T en función de la pregunta realizada. Esto podría relacionarse con la posible carencia de una idea global del fenómeno tecnológico. Es decir, confunden las visiones de un mismo objeto desde distintos ángulos con visiones de distintos objetos.

Observamos una falta de los componentes esenciales de la alfabetización en cyt en alumnos que han elegido una carrera tecnológica (Biotecnología, Ingeniería en Alimentos). Por ejemplo, ya que se trata de la formación profesional, cabe suponer que sus implicancias, сут para la sociedad estarían más presentes.

Científicos, educadores y organizaciones internacionales de educación en ciencias coinciden en que todos los estudiantes desarrollen concepciones informadas y apropiadas sobre la Nd CyT. El presente diagnóstico señala la necesidad de una mejor y más amplia (un 
curso aislado no es suficiente) alfabetización en Ciencia, en Tecnología y en sus vínculos, tanto en el nivel educativo previo (educación media) como desde el comienzo de una carrera universitaria tecnológica.

Este análisis justifica con creces el diseño de una didáctica orientada a la EeT que construya, junto con los estudiantes, un modelo complejo de T, que dé cuenta del actual conocimiento filosófico, sociológico, económico, ingenieril que se tiene de la misma. Esto es, promover una Eet consciente y responsable de la orientación de su función educativa para luego elaborar propuestas prácticas que permitan alcanzar esas finalidades (Gordillo y Galbarte, 2002).

Finalmente, unas observaciones relacionadas al instrumento de medición utilizado: el mismo mostró ventajas en cuanto a su practicidad de aplicación, teniendo en cuenta también el amplio número de trabajos previos que lo respaldan. Otra ventaja fue la posibilidad del tratamiento de datos de forma estadística. También mostró desventajas, por no ser un instrumento desarrollado específicamente para evaluar el concepto de Tecnología, algunos aspectos no están representados. Además, la división del tema en cuestiones parciales impide que los estudiantes puedan expresar una visión integral del fenómeno. Es decir, resulta difícil conocer si han de poseer un modelo mental complejo. De esto se deduce la necesidad de suplementar el mismo con uno o varios instrumentos que permitan a los estudiantes expresar la complejidad del modelo mental. En cuanto a los aportes a la metodología de evaluación, se ha mostrado que el análisis no estadístico a partir de NDA permite observar cambios al interior de la población que quedan ocultos con el método estadístico. Si bien el método empleado es no estadístico, es un llamado de atención para desarrollar herramientas de esta índole que puedan detectar el tipo de variaciones mencionadas. También resultó útil para complementar el método que detecta variaciones estadísticamente significativas, ya que evidenció, de qué forma cambió la media de la opinión de los estudiantes y en qué nivel se afecta con los tratamientos.

\section{Agradecimientos}

Proyecto EDU 201 5-64642-R (AEI/FEDER, UE) financiado por la Agencia Estatal de Investigación (AEl) y el Fondo Europeo de Desarrollo Regional (FEDER).

Proyecto PICT 2014-1 134, financiado por ANPCYT, Agencia Nacional de Promoción Científica y Tecnológica, Argentina.

CONICET, Consejo Nacional de Investigaciones Científicas y Técnicas, Argentina.

\section{Referencias}

Acevedo Díaz, J. A., Vasquez Alonso, A., Manassero Mas, M. A. y Acevedo Romero, P. (2005). Aplicación de una nueva metodología para evaluar las creencias del profesorado sobre la tecnología y sus relaciones con la ciencia. Educación Química, 16(3), 372-382. 
Barak, M. y Zadok, Y. (2009). Robotics projects and learning concepts in science, technology and problem solving. International Journal of Technology and Design Education, 19(3), 289-307. https://doi.org/10.1007/ s 10798-007-9043-3

Bennássar, A., Vázquez, A., Manassero M. A. y García-Carmona, A. (Coords.) (2010). Ciencia, tecnología y sociedad en Iberoamérica. OEl.

Bortz, G. y Thomas, H. (2019). Parasites, bugs and banks: problems and constraints of designing policies and technologies that transform R\&D into healthcare solutions: the case of Chagas disease in Argentina (2007-2017). Innovation and Development, 1-19. https://doi.org/10.1080/2 157930X.2019.1567904

Cadena, L. F. G. y Agudelo, E. O. M. (2013). La naturaleza de la ciencia y la tecnología en instructores de educación técnica y tecnológica. Educación y Territorio, 3(1), 67-87.

Cajas, F. (200la). The science/technology interaction: Implications for science literacy. Journal of Research in Science Teaching: The Official Journal of the National Association for Research in Science Teaching, 38(7), 715-729.

Cajas, F. (2001b). Alfabetización científica y tecnológica: la transposición didáctica del conocimiento tecnológico. Enseñanza de las ciencias: revista de investigación y experiencias didácticas, 19(2), 243-254.

Calderon Medina, L. B., Ceron Alarcon, D. F. y Gomez Amaya, J. (2015). Diseño de un ambiente virtual de aprendizaje por medio del cual se comprenda y apropie el concepto de tecnología en los estudiantes de grado sexto del Colegio Paulo vI IED [Tesis de especialización, Corporación Universitaria Minuto de Dios]. Repositorio institucional Uniminuto http://hdl.handle. net/10656/3055
Campo, V. M. G. (2003). ¿ Educación tecnológica o formación profesional?: El caso de Colombia. Revista de la educación superior, 32(126), 4.

Cardwell, D. (1994). The Fontana History of Technology. London: Harper Collins Publishers. Traducción castellana (1996): Historia de la Tecnología. Alianza.

De Vries, M. J. (2018). Technology education: An international history. Handbook of Technology Education, (pp. 73-84). Springer International Publishing

Díaz, J. A. A. (1995). Educación tecnológica desde una perspectiva CTS. Una breve revisión del tema. Alambique. Didáctica de las Ciencias Experimentales, 3, 75-84.

Díaz, J. A. A., Alonso, Á. V., Antonia, M., Mas, M. y Romero, P. A. (2003). Creencias sobre la tecnología y sus relaciones con la ciencia. Revista electrónica de enseñanza de las ciencias, 2(3), 353-376.

Férnandez, I., Gil, D., Vilches, A., Valdés, P., Cachapuz, A., Praia, J. y Salinas, J. (2003). El olvido de la tecnología como refuerzo de las visiones deformadas de la ciencia. Revista electrónica de enseñanza de las ciencias, 2(3), 33 1-352.

Ferreira-Gauchía, C., Vilches, A. y Gil-Pérez, D. (2012). Concepciones acerca de la naturaleza de la tecnología y de las relaciones Ciencia, Tecnología, Sociedad y Ambiente en la educación tecnológica. Enseñanza de las ciencias: revista de investigación y experiencias didácticas, 30(2), 197-218.

Fressoli, M. (2015). Movimientos de base y desarrollo sustentable: La construcción de caminos alternativos. Cienc Investig, 65, 55-68.

Gauchía, C. F., Pérez, D. G. y Vilches, A. (2013). Imagen de la Tecnología transmitida por los textos de educación tecnológica. http://www.oei.es/decada/. Contribución a la Década de la Educación para el Desarrollo Sostenible, Naciones Unidas (2005-2014). 
Gilbert, J. K. (1995). Educación tecnológica: una nueva asignatura en todo el mundo. Enseñanza de las ciencias: revista de investigación y experiencias didácticas, 13(1), 15-24.

González Ramírez, P. (2017). Representaciones sociales sobre el concepto de tecnología de los estudiantes de la licenciatura de diseño tecnológico de la Universidad Pedagógica Nacional. Colección Licenciatura en Diseño tecnológico. http:/ /hdl.handle.net/20.500.12209/7693

Gordillo, M. M. y Galbarte, J. C. G. (2002). Reflexiones sobre la educación tecnológica desde el enfoque CTS. Revista Iberoamericana de educación, 28(1).

Hugo, D., Olavegogeasoechea, M., Orlandini, L., Salica, M. y Ávila, S. (2014). Investigar e innovar la formación cts inicial de profesores de ciencias aplicando una secuencia de enseñanza-aprendizaje sobre las decisiones tecnológicas. Uni-pluriversidad, 14(2), 72-80.

Hugo, D. V., Olea, P., Olavegogeasoechea, M., Mariel, F. N. y Ávila, S. (2012). Tecnología: Mucho más que aparatos. Revista de Ensino de Ciências e Matemática, 3(3), 105-1 15.

Layton, D. (1988). Revaluing the T in STs. International Journal of Science Education, 10(4), 367-378.

Lewis, T. (1991). Introducing technology into school curricula. J. Curriculum Studies, 23(2), 141- 154.

Lewis, T. (2000). Technology education and developing countries. International Journal of Technology and Design Education, 10(2), 163-179.

Londoño, E. A. (1991). Marco para una discusión sobre educación: la ciencia y la tecnología, aspectos claves de la soberanía nacional. Tecné Episteme y Didaxis: TED, (1). https://doi.org/10.17227/ted.num 1-5718

Maiztegui, A., Acevedo, J.A., Caamaño, A., Cachapuz, A., Cañal, P., Carvalho, A.M.P., Del Carmen, L., Dumas Carré, A., Garritz, A., Gil, D., González, E., Gras-Martí, A., Guisasola, J., López-Cerezo J.A., Macedo, B., Martínez-Torregrosa, J., Moreno, A., Praia, J., Rueda, C., Tricárico, H., Valdés, P. y Vilches, A. (2002). Papel de la tecnología en la educación científica: una dimensión olvidada. Revista Iberoamericana de Educación, 28, 129-155.

MINCYT. (2012). Plan Nacional de Ciencia, Tecnología e Innovación Argentina Innovadora 2020. https://www.argentina.gob.ar/sites/default/files/pai2020.pdf

Molina, F. T. (201 1). Consumo tecnológico y educación tecnológica: fundamentos filosóficos para un proyecto futuro. Sociologias, 13(26), 154-175.

Pacey, A. (1983). The culture of technology. Massachusetts: MIT press.

Pearce, J. M. (2017). Impacts of Open Source. Hardware in Science and Engineering. Bridge, 47, 24-31.

Smith, A., Ely, A., Fressoli, M., Abrol, D. y Arond, E. (2016). Grassroots innovation movements. New York: Routledge. Taylor and Francis Group.

Tessio, N. M., Di Stefano, A. y Porro, S. ¿Políticas Tecnológicas? Memoria 2008 UNQ. Argentina. 
UNCTAD. (2017). New innovation approaches to support the implementation of The sustainable development goals. New York and Geneva: United Nations. http://unctad.org/en/ Publicationslibrary/dtlstict2017d4_en.pdf

Valdés, P., Valdés, R., Guisasola, J. y Santos, T. (2002). Implicaciones de las relaciones ciencia-tecnología en la educación científica. Revista Iberoamericana de Educación, 28(1).
Vázquez Alonso, A., Acevedo Díaz, J. A. y Manassero Mas, M.A. (2006). Aplicación del cuestionario de opiniones CTS con una nueva metodología en la evaluación de un curso de formación CTs del profesorado. Tarbiya, Revista de Investigación e Innovación Educativa, 37, 31-65.

\section{Para citar este artículo}

Veiga, L. y Roncaglia, D. (2020). Concepciones acerca de la Tecnología de estudiantes nóveles de carreras científico-tecnológicas en Argentina. Tecné, Episteme y Didaxis: TED, (48), $111-125$. https://doi.org/10.17227/ted. num48-10933

- 